\title{
A New Skew-normal Density
}

\author{
Maryam Sharafi ${ }^{\dagger}$ and Javad Behboodian ${ }^{\ddagger, *}$ \\ $\dagger$ Shiraz University \\ ‡ Islamic Azad University, Shiraz Branch
}

\begin{abstract}
We present a new skew-normal distribution, denoted by $\operatorname{NSN}(\lambda)$. We first derive the density and moment generating function of $\operatorname{NSN}(\lambda)$. The properties of $\operatorname{SN}(\lambda)$, the known skewnormal distribution of Azzalini, and $\operatorname{NSN}(\lambda)$ are compared with each other. Finally, a numerical example for testing about the parameter $\lambda$ in $\operatorname{NSN}(\lambda)$ is given.
\end{abstract}

Keywords. skew-normal distribution; a new skew-normal distribution; moment generating function; skewness; kurtosis; testing hypothesis.

\section{Introduction}

Azzalini (1985) introduced the following density function by the name of skewnormal density with parameter $\lambda$,

$$
\varphi(z ; \lambda)=2 \varphi(z) \Phi(\lambda z), \quad z \in \mathbb{R}, \quad \lambda \in \mathbb{R}
$$

where $\varphi(z)$ and $\Phi(z)$ are density and distribution functions of standard normal random variable, respectively. We denote a random variable $Z_{\lambda}$ with the above density by $Z_{\lambda} \sim \mathrm{SN}(\lambda)$. This density and its generalization have been studied during the past years. For example, the distribution $\operatorname{GSN}(\lambda)$, given by Gupta and Gupta (2004), is a useful generalization of $\mathrm{SN}(\lambda)$.

* Corresponding author 
In this paper we consider the density

$$
f(x ; \lambda)=c(\lambda) \varphi(x) \Phi^{2}(\lambda x),
$$

where $c(\lambda)$ is given by

$$
c(\lambda)=\frac{1}{E\left\{\Phi^{2}(\lambda U)\right\}},
$$

with $U \sim \mathrm{N}(0,1)$. We denote a random variable with this new density by $X_{\lambda} \sim \operatorname{NSN}(\lambda)$, which is in fact a special case of $\operatorname{GSN}(\lambda)$. Azzalini (1985) showed that the maximum value of skewness for $\operatorname{SN}(\lambda)$ is about 0.995 . The motivation of introducing this new density is the fact that it has a bigger skewness.

In section 2 , we use orthant probability to compute $c(\lambda)$ easily without integration and illustrate a real data. Section 3 presents two representation theorems regarding the properties of $\operatorname{NSN}(\lambda)$. The moment generating function, some moments, skewness, and kurtosis of $\operatorname{NSN}(\lambda)$ are given in section 4. In section 5 , we compare the properties of $\operatorname{SN}(\lambda)$ and $\operatorname{NSN}(\lambda)$. Finally, in section 6 , we generate some data from $\operatorname{NSN}(\lambda)$ and we concentrate on a testing about the parameter $\lambda$.

\section{Calculation of $\mathrm{c}(\boldsymbol{\lambda})$ by Orthant Probability}

An orthant probability is the probability $P\left(V_{1}>0, V_{2}>0, \ldots, V_{n}>0\right)$ where $\mathbf{V}=\left(V_{1}, V_{2}, \ldots, V_{n}\right)$ is a multivariate normal vector with mean 0 and covariance matrix $\boldsymbol{\Sigma}=\left(\rho_{i j}\right)$, where $\rho_{i i}=1$ and $\rho_{i j}=\operatorname{cov}\left(V_{i}, V_{j}\right), i, j=1,2, \ldots, n$ (see Kotz et al., 2000).

Now, we write

$$
\begin{aligned}
E\left\{\Phi^{2}(\lambda U)\right\} & =\int_{-\infty}^{\infty} \Phi^{2}(\lambda u) \varphi(u) d u \\
& =\int_{-\infty}^{\infty} P\left(U_{1} \leqslant \lambda u, U_{2} \leqslant \lambda u\right) \varphi(u) d u,
\end{aligned}
$$

where $U_{1}$ and $U_{2}$ are i.i.d. $\mathrm{N}(0,1)$ and independent from $U \sim \mathrm{N}(0,1)$. Using the above integral we have

$$
\begin{aligned}
E\left\{\Phi^{2}(\lambda U)\right\} & =\int_{-\infty}^{\infty} P\left(U_{1} \leqslant \lambda U, U_{2} \leqslant \lambda U \mid U=u\right) \varphi(u) d u \\
& =P\left(U_{1} \leqslant \lambda U, U_{2} \leqslant \lambda U\right) \\
& =P\left(V_{1} \geqslant 0, V_{2} \geqslant 0\right),
\end{aligned}
$$


where

$$
V_{1}=\frac{\lambda U-U_{1}}{\sqrt{1+\lambda^{2}}}, \quad V_{2}=\frac{\lambda U-U_{2}}{\sqrt{1+\lambda^{2}}}
$$

with

$$
\left(V_{1}, V_{2}\right) \sim \mathrm{N}_{2}(\mathbf{0}, \boldsymbol{\Sigma}), \quad \boldsymbol{\Sigma}=\left(\begin{array}{cc}
1 & \frac{\lambda^{2}}{1+\lambda^{2}} \\
\frac{\lambda^{2}}{1+\lambda^{2}} & 1
\end{array}\right) .
$$

Hence, we have

$$
E\left\{\Phi^{2}(\lambda U)\right\}=\frac{1}{4}+\frac{1}{2 \pi} \sin ^{-1}\left(\frac{\lambda^{2}}{1+\lambda^{2}}\right) .
$$

Using the simple trigonometric relation,

$$
\frac{1}{4}+\frac{1}{2 \pi} \sin ^{-1} x=\frac{1}{\pi} \tan ^{-1} \sqrt{\frac{1+x}{1-x}}, \quad-1<x<1
$$

from (2), we obtain

$$
c(\lambda)=\frac{1}{E\left\{\Phi^{2}(\lambda U)\right\}}=\frac{\pi}{\tan ^{-1} \sqrt{1+2 \lambda^{2}}} .
$$

This coefficient is obtained in Arnold et al. (2002) by integration, but our method, by orthant probability, is much easier.

Therefore, density (1) becomes

$$
f(x ; \lambda)=\frac{\pi}{\tan ^{-1} \sqrt{1+2 \lambda^{2}}} \varphi(x) \Phi^{2}(\lambda x) .
$$

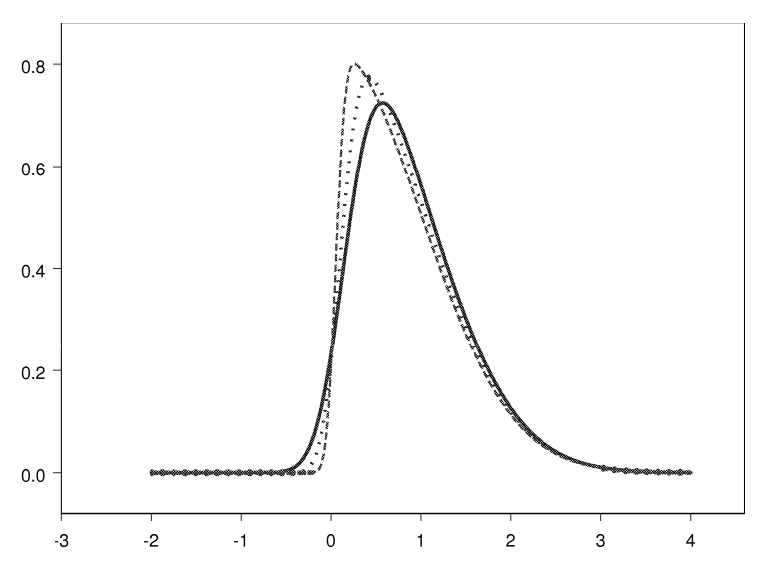

Figure 1. The density of $\operatorname{NSN}(\lambda)$ for $\lambda=3$ (soiled line), $\lambda=5$ (dotted line), and $\lambda=10$ (dashed line). 
Table 1. MLE's parameters for IQ score data

\begin{tabular}{cccc}
\hline \hline Distribution & $\mathbf{N}\left(\boldsymbol{\mu}, \boldsymbol{\sigma}^{\mathbf{2}}\right)$ & $\mathbf{S N}(\boldsymbol{\mu}, \boldsymbol{\sigma}, \boldsymbol{\lambda})$ & $\mathbf{N S N}(\boldsymbol{\mu}, \boldsymbol{\sigma}, \boldsymbol{\lambda})$ \\
\hline$\widehat{\mu}$ & 106.653 & 98.79 & 94.88 \\
$\widehat{\sigma}$ & 8.23 & 11.38 & 13.07 \\
$\widehat{\lambda}$ & - & 1.71 & 2.09 \\
Log-likelihood & -183.387 & -182.436 & -182.206 \\
\hline
\end{tabular}

Figure 1 shows the shape of $f(x ; \lambda)$ for some values of $\lambda$.

The location-scale of $\operatorname{NSN}(\lambda)$ is defined as that of $Y=\mu+\sigma X_{\lambda}$, where $\mu \in \mathbb{R}, \sigma>0$, and $X_{\lambda} \sim \operatorname{NSN}(\lambda)$. Its density is given by

$$
f(y ; \theta)=\left(\frac{\pi}{\sigma \tan ^{-1} \sqrt{1+2 \lambda^{2}}}\right) \varphi\left(\frac{y-\mu}{\sigma}\right) \Phi^{2}\left(\lambda \cdot \frac{y-\mu}{\sigma}\right),
$$

where $\theta=(\mu, \sigma, \lambda)$. We denote this by $Y \sim \operatorname{NSN}(\mu, \sigma, \lambda)$.

We now fit this new distribution on the real IQ score data for 52 non-white males given by Gupta and Brown (2001). Table 1 and Figure 2 show that our distribution better fits the data comparing with the normal and Azzalini's distributions.

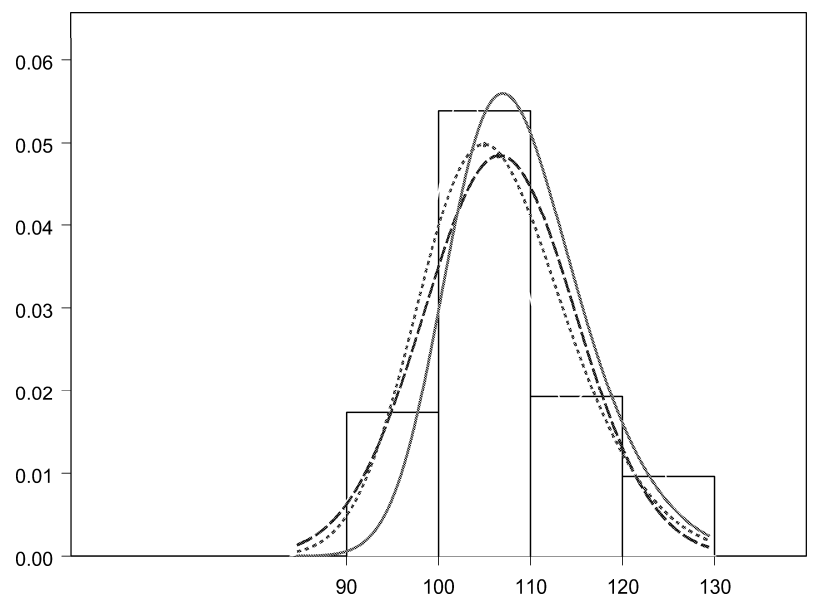

Figure 2. Histogram of 52 Otis IQ Scores. The lines represent distributions fitted using maximum likelihood estimation: $\mathrm{N}\left(\hat{\mu}, \hat{\sigma}^{2}\right)$ (dotted line), $\operatorname{SN}(\hat{\mu}, \hat{\sigma}, \hat{\lambda})$ (dashed line), and $\operatorname{NSN}(\hat{\mu}, \hat{\sigma}, \hat{\lambda})$ (soiled line). 


\section{Two Representation Theorems about $\operatorname{NSN}(\lambda)$}

Azzalini and Dalla-Valle (1996) define the density of the bivariate skew-normal vector $\left(Y_{1}, Y_{2}\right)^{\mathrm{T}} \sim \mathrm{SN}_{2}(\boldsymbol{\Omega}, \boldsymbol{\alpha})$ by

$$
\varphi_{2}\left(y_{1}, y_{2} ; \rho, \boldsymbol{\alpha}\right)=2 \varphi\left(y_{1}, y_{2} ; \rho\right) \Phi\left(\alpha_{1} y_{1}+\alpha_{2} y_{2}\right),
$$

where $\varphi\left(y_{1}, y_{2} ; \rho\right)$ is the standard bivariate normal density with

$$
\boldsymbol{\Omega}=\left(\begin{array}{ll}
1 & \rho \\
\rho & 1
\end{array}\right), \quad|\rho|<1,
$$

and

$$
\boldsymbol{\alpha}=\left(\begin{array}{c}
\alpha_{1} \\
\alpha_{2}
\end{array}\right) .
$$

By the following representation theorems, we relate $X_{\lambda}$ with normal and bivariate skew-normal variables.

Theorem 1 (first representation theorem). If $\left(Y_{1}, Y_{2}\right)^{\mathrm{T}} \sim \mathrm{SN}_{2}(\boldsymbol{\Omega}, \boldsymbol{\alpha})$ with $\alpha=\left(\rho / \sqrt{1-\rho^{2}}, 0\right)^{\mathrm{T}}$, then

$$
\left(Y_{1} \mid Y_{2}>0\right) \stackrel{\mathrm{D}}{=} X_{\lambda} \sim \operatorname{NSN}(\lambda), \quad \lambda=\frac{\rho}{\sqrt{1-\rho^{2}}}
$$

where $\stackrel{\underline{D}}{=}$ denotes equality in distribution.

\section{Proof.}

$$
\begin{aligned}
P\left(Y_{1} \leqslant x \mid Y_{2}>0\right) & =\frac{P\left(Y_{1} \leqslant x, Y_{2}>0\right)}{P\left(Y_{2}>0\right)} \\
& =\frac{2 \int_{-\infty}^{x} \int_{0}^{\infty} \varphi\left(y_{1}, y_{2} ; \rho\right) \Phi\left(\lambda y_{1}\right) d y_{2} d y_{1}}{P\left(Y_{2}>0\right)} \\
& =\frac{2 \int_{-\infty}^{x} \varphi\left(y_{1}\right) \Phi^{2}\left(\lambda y_{1}\right) d y_{1}}{P\left(Y_{2}>0\right)} .
\end{aligned}
$$

On the other hand, by Azzalini and Capitanio (1999),

$$
Y_{2} \sim \mathrm{SN}\left(\frac{\lambda^{2}}{\sqrt{1+2 \lambda^{2}}}\right),
$$

and by Gupta and Brown (2001),

$$
P\left(Y_{2}>0\right)=\frac{1}{2}+\frac{1}{\pi} \tan ^{-1} \frac{\lambda^{2}}{\sqrt{1+2 \lambda^{2}}} .
$$


Now, using the following trigonometric relation for $x \in \mathbb{R}$,

$$
\frac{1}{2}+\frac{1}{\pi} \tan ^{-1} \frac{x^{2}}{\sqrt{1+2 x^{2}}}=\frac{2}{\pi} \tan ^{-1} \sqrt{1+2 x^{2}},
$$

we have

$$
P\left(Y_{2}>0\right)=\frac{2}{\pi} \tan ^{-1} \sqrt{1+2 \lambda^{2}} .
$$

From (5) and (6), we obtain

$$
P\left(Y_{1} \leqslant x \mid Y_{2}>0\right)=\int_{-\infty}^{x} \frac{\pi}{\tan ^{-1} \sqrt{1+2 \lambda^{2}}} \varphi\left(y_{1}\right) \Phi^{2}\left(\lambda y_{1}\right) d y_{1},
$$

which is the distribution of $X_{\lambda}$ by (4). This completes the proof.

Theorem 2 (second representation theorem). If $\left(U_{1}, U_{2}, U_{3}\right) \sim \mathrm{N}_{3}(\mathbf{0}, \boldsymbol{\Sigma})$ with

$$
\boldsymbol{\Sigma}=\left(\begin{array}{ccc}
1 & \rho & \rho \\
\rho & 1 & \rho^{2} \\
\rho & \rho^{2} & 1
\end{array}\right)
$$

then

$$
\left(U_{1} \mid \min \left\{U_{2}, U_{3}\right\}>0\right) \stackrel{\mathrm{D}}{=} X_{\lambda} \sim \mathrm{NSN}(\lambda), \quad \lambda=\frac{\rho}{\sqrt{1-\rho^{2}}} .
$$

Proof. Let $U=\min \left\{U_{2}, U_{3}\right\}$ and compute the joint density of $U_{1}$ and $U$. Using the fact that the pair $\left(U_{2}, U_{3}\right)$ is exchangeable and $\left(U_{1}, U_{2}\right) \stackrel{\mathrm{D}}{=}\left(U_{1}, U_{3}\right)$, we have

$$
\begin{aligned}
P\left(U_{1} \leqslant u_{1}, U \leqslant u_{2}\right)= & P\left(U_{1} \leqslant u_{1}, U_{2} \leqslant u_{2} \mid U_{2} \leqslant U_{3}\right) P\left(U_{2} \leqslant U_{3}\right) \\
& +P\left(U_{1} \leqslant u_{1}, U_{3} \leqslant u_{2} \mid U_{3} \leqslant U_{2}\right) P\left(U_{3} \leqslant U_{2}\right) \\
= & P\left(U_{1} \leqslant u_{1}, U_{2} \leqslant u_{2} \mid U_{0} \geqslant 0\right)
\end{aligned}
$$

where

$$
U_{0}=\frac{U_{3}-U_{2}}{\sqrt{2\left(1-\rho^{2}\right)}} .
$$

On the other hand, $\left(U_{0}, U_{1}, U_{2}\right) \sim \mathrm{N}_{3}\left(\mathbf{0}, \boldsymbol{\Sigma}^{*}\right)$ with

$$
\boldsymbol{\Sigma}^{*}=\left(\begin{array}{ccc}
1 & 0 & -\sqrt{\frac{1-\rho^{2}}{2}} \\
0 & 1 & \rho \\
-\sqrt{\frac{1-\rho^{2}}{2}} & \rho & 1
\end{array}\right)
$$


By Azzalini and Dalla-Valle (1996)

$$
\left(\left(U_{1}, U_{2}\right) \mid U_{0}>0\right) \sim \mathrm{SN}_{2}(\boldsymbol{\Omega}, \boldsymbol{\alpha}),
$$

where

$$
\boldsymbol{\Omega}=\left(\begin{array}{ll}
1 & \rho \\
\rho & 1
\end{array}\right), \quad \boldsymbol{\alpha}=\left(\begin{array}{c}
\frac{\rho}{\sqrt{1-\rho^{2}}} \\
\frac{-1}{\sqrt{1-\rho^{2}}}
\end{array}\right) .
$$

Now, from (8) and (9) we have

$$
\left(U_{1}, U\right) \sim \mathrm{SN}_{2}(\Omega, \alpha)
$$

Then, by the approach of Theorem 1, we conclude that

$$
\left(U_{1} \mid \min \left\{U_{2}, U_{3}\right\}>0\right) \stackrel{\mathrm{D}}{=} X_{\lambda} .
$$

\section{Moment Generating Function of $\operatorname{NSN}(\lambda)$}

In this section, we find the moment generating function (m.g.f.) of $X_{\lambda}$ which has density (4).

Theorem 3 The m.g.f. of $X_{\lambda}$ is

$$
M_{X_{\lambda}}(t)=\frac{\pi}{\tan ^{-1} \sqrt{1+2 \lambda^{2}}} \exp \left\{\frac{t^{2}}{2}\right\} \Phi_{1}\left(\frac{\lambda t}{\sqrt{1+\lambda^{2}}} ; \frac{1}{\sqrt{1+2 \lambda^{2}}}\right),
$$

where $\Phi_{1}(z ; \theta)$ is the distribution function of $Z_{\theta} \sim \mathrm{SN}(\theta)$, given by Azzalini (1985), as follows.

$$
\Phi_{1}(z ; \theta)=\Phi(z)-2 \int_{z}^{\infty} \int_{0}^{\theta w} \varphi(u) \varphi(w) d u d w .
$$

Proof. Using density (4) and the change of variable $x-t=u$, we have

$$
\begin{aligned}
M_{X_{\lambda}}(t)=E\left(\exp \left\{t X_{\lambda}\right\}\right) & =\int_{-\infty}^{\infty} \frac{\pi}{\tan ^{-1} \sqrt{1+2 \lambda^{2}}} \exp \{t x\} \varphi(x) \Phi^{2}(\lambda x) d x \\
& =\frac{\pi}{\tan ^{-1} \sqrt{1+2 \lambda^{2}}} \exp \left\{\frac{t^{2}}{2}\right\} E\left\{\Phi^{2}(\lambda U+\lambda t)\right\},
\end{aligned}
$$

with $U \sim \mathrm{N}(0,1)$. We can show that

$$
E\left\{\Phi^{2}(\lambda U+\lambda t)\right\}=\Phi_{1}\left(\frac{\lambda t}{\sqrt{1+\lambda^{2}}} ; \frac{1}{\sqrt{1+2 \lambda^{2}}}\right)
$$

(see Appendix A1). Therefore, we have (10). 


\subsection{Moments of $\mathrm{X}_{\lambda}$}

We can find expectation, variance, and third and forth moments of $X_{\lambda}$ by taking derivative from $M_{X_{\lambda}}(t)$ with respect to $t$. The results are

$$
\begin{gathered}
\mu_{\lambda}=E\left(X_{\lambda}\right)=\frac{\pi}{\tan ^{-1} \sqrt{1+2 \lambda^{2}}} \cdot \frac{\lambda}{\sqrt{2 \pi} \sqrt{1+\lambda^{2}}}, \\
\sigma_{\lambda}^{2}=\operatorname{var}\left(X_{\lambda}\right)=1+\frac{\lambda^{2}}{\left(1+\lambda^{2}\right) \sqrt{1+2 \lambda^{2}} \tan ^{-1} \sqrt{1+2 \lambda^{2}}}-\mu_{\lambda}^{2} .
\end{gathered}
$$

To find skewness and kurtosis of $X_{\lambda}$, we have

$$
\begin{gathered}
E\left(X_{\lambda}^{3}\right)=\left(\frac{3+2 \lambda^{2}}{1+\lambda^{2}}\right) \mu_{\lambda}, \\
E\left(X_{\lambda}^{4}\right)=3 E\left(X_{\lambda}^{2}\right)+\frac{\lambda^{2}\left(5 \lambda^{2}+3\right)}{\left(1+\lambda^{2}\right)^{2}\left(1+2 \lambda^{2}\right)^{\frac{3}{2}} \tan ^{-1} \sqrt{1+2 \lambda^{2}}}
\end{gathered}
$$

(see Appendix A2). Using (12)-(15) and some computation we obtain

$$
\begin{gathered}
3<\text { kurtosis of } X_{\lambda}=\frac{E\left(X_{\lambda}-\mu_{\lambda}\right)^{4}}{\sigma_{\lambda}^{4}}<3.8692 \\
-5.6330<\text { skewness of } X_{\lambda}=\frac{E\left(X_{\lambda}-\mu_{\lambda}\right)^{3}}{\sigma_{\lambda}^{3}}<5.6330
\end{gathered}
$$

We may consider the more general skew-normal density

$$
f(x ; \lambda)=c(\lambda) \varphi(x) \Phi^{n}(\lambda x), \quad n=1,2, \ldots
$$

by the name of skew-normal density of order $n$ with distribution $\operatorname{GSN}(\lambda)$, which was introduced by Gupta and Gupta (2004). More Properties of this density are studied by Sharafi and Behboodian (2006). The result of Theorem 3 can also be obtained from this general density.

\section{Comparison of $\operatorname{SN}(\lambda)$ and $\operatorname{NSN}(\lambda)$}

In this section, we discuss some properties of $Z_{\lambda} \sim \operatorname{SN}(\lambda)$ and $X_{\lambda} \sim \operatorname{NSN}(\lambda)$.

1. For $\lambda=0, X_{\lambda} \stackrel{\mathrm{D}}{=} Z_{\lambda} \sim \mathrm{N}(0,1)$.

2. As $\lambda \rightarrow \pm \infty$, the densities of $X_{\lambda}$ and $Z_{\lambda}$ go to the half-normal density, i.e., the density of $|U|$ with $U \sim \mathrm{N}(0,1)$. 
3. $-X_{\lambda} \sim \operatorname{NSN}(-\lambda)$ and $-Z_{\lambda} \sim \operatorname{SN}(-\lambda)$.

4. $X_{\lambda}$ and $Z_{\lambda}$ are both strongly unimodal (see Karlin, 1968).

5. Skewness of $X_{\lambda}$ and $Z_{\lambda}$ is positive for $\lambda>0$ and negative for $\lambda<0$.

6. $E\left(X_{\lambda}^{k}\right) \geqslant E\left(Z_{\lambda}^{k}\right) \geqslant 0$ for $\lambda \geqslant 0, \quad k=1,3$

$E\left(X_{\lambda}^{k}\right) \leqslant E\left(Z_{\lambda}^{k}\right) \leqslant 0 \quad$ for $\quad \lambda \leqslant 0, \quad k=1,3$.

7. $E\left(X_{\lambda}^{2}\right) \geqslant E\left(Z_{\lambda}^{2}\right)$ for $\lambda \in \mathbb{R}$.

8. $\operatorname{var}\left(X_{\lambda}\right) \leqslant \operatorname{var}\left(Z_{\lambda}\right) \quad$ for $\quad \lambda \in \mathbb{R}$.

9. skewness of $X_{\lambda} \geqslant$ skewness of $Z_{\lambda} \geqslant 0$ for $\lambda \geqslant 0$ skewness of $X_{\lambda} \leqslant$ skewness of $Z_{\lambda} \leqslant 0$ for $\lambda \leqslant 0$.

10. kurtosis of $X_{\lambda} \geqslant$ kurtosis of $Z_{\lambda}$ for $\lambda \in \mathbb{R}$.

11. $P\left(X_{\lambda} \geqslant 0\right) \geqslant P\left(Z_{\lambda} \geqslant 0\right)$ for $\lambda \geqslant 0$ $P\left(X_{\lambda} \geqslant 0\right) \leqslant P\left(Z_{\lambda} \geqslant 0\right)$ for $\lambda \leqslant 0$.

(see Appendix A3).

\section{Hypothesis Testing about $\lambda$}

Let $X_{1}, X_{2}, \ldots, X_{n}$ be i.i.d. from $\operatorname{NSN}(\lambda)$. We want to test $H_{0}: \lambda=\lambda_{0}$ versus $H_{1}: \lambda \neq \lambda_{0}$. It is easy to show that

$$
T=\frac{1}{n} \sum_{i=1}^{n} \frac{X_{i}}{\Phi\left(\lambda X_{i}\right)}
$$

is a decreasing function of $\lambda$. Using

$$
E\left(Z_{\lambda}\right)=\sqrt{\frac{2}{\pi}}\left(\frac{\lambda}{\sqrt{1+\lambda^{2}}}\right)
$$

(see Azzalini, 1985), we have

$$
\begin{gathered}
\mu_{T}=E(T)=\sqrt{\frac{\pi}{2}}\left(\frac{\lambda}{\sqrt{1+\lambda^{2}}}\right)\left(\frac{1}{\tan ^{-1} \sqrt{1+2 \lambda^{2}}}\right), \\
\sigma_{T}^{2}=\operatorname{var}(T)=\frac{\pi}{n \tan ^{-1} \sqrt{1+2 \lambda^{2}}}\left(1-\frac{\lambda^{2}}{2\left(1+\lambda^{2}\right) \tan ^{-1} \sqrt{1+2 \lambda^{2}}}\right) .
\end{gathered}
$$


(see Appendix A4). For large $n$, we have approximately (under $H_{0}$ )

$$
Z=\frac{T-\mu_{T}}{\sigma_{T}} \sim \mathrm{N}(0,1) .
$$

Therefore, the critical region for the above test at the level $\alpha$ is $|Z|>z_{\alpha / 2}$, where $P\left(Z>z_{\alpha / 2}\right)=\alpha / 2$.

We observe that this statistic is simpler than the similar statistic used by Gupta and Gupta (2004). Using the following generated data from NSN(1) with size 60 , we want to test $H_{0}: \lambda=1$ versus $H_{1}: \lambda \neq 1$. Under $H_{0}$ we obtain

$$
\begin{gathered}
t=0.96331, \quad \mu_{T}=0.846284, \quad \sigma_{T}=0.195198 \\
z=0.599830, \quad p \text {-value }=2 P(Z>0.599830)=0.54862
\end{gathered}
$$

Therefore $H_{0}$ is not rejected, and the power of the test for $\lambda=0$ is 0.9638 at level $\alpha=0.05$.

$\begin{array}{rrrrrr}1.01597 & 1.16863 & 0.29148 & 1.00454 & 0.29135 & 1.80183 \\ 0.44544 & -0.00673 & 1.81410 & -0.57200 & 1.47283 & 0.04330 \\ 2.46565 & -0.03888 & 1.13157 & 0.44446 & 0.64613 & 1.13833 \\ 1.49379 & 0.00786 & 0.54528 & 2.12881 & 1.58779 & 1.32810 \\ 2.05071 & 0.56590 & 0.70947 & 1.60860 & 1.70980 & 1.36679 \\ 1.19152 & -0.01076 & -0.19823 & 0.58204 & -0.02293 & 0.27317 \\ 1.96351 & -0.09878 & 0.46880 & 1.60463 & 0.48174 & 1.48968 \\ 0.68240 & 1.46606 & 0.61545 & 1.46024 & 0.56457 & 1.53633 \\ 0.10162 & 0.14067 & 0.48807 & 0.59445 & 0.58263 & 1.01765 \\ 0.45428 & 0.89725 & 2.09898 & 0.22074 & 0.18980 & 1.43234\end{array}$

\section{Acknowledgement}

The authors sincerely thank the editor and referees for their valuable comments and suggestions.

\section{References}


Arnold, B.C.; Beaver, R.J. (2002). Skewed multivariate models related to hidden truncation and/or selective reporting (with discussion). Test 11, 7-54

Azzalini, A. (1985). A class of distributions with includes the normal ones. Scand. J. Statist. 12, 171-178.

Azzalini, A. (1986). Further results on a class of distribution which includes the normal ones. Statistica 46, 199-208.

Azzalini, A.; Capitanio, A. (1999). Statitical applications of the multivarite skew-normal distributions. J. R. Stat. Soc. Ser. B Stat. Methodol. 61, 579-602.

Azzalini, A.; Dalla-Valle, A. (1996). The multivarite skew-normal distribution, Biometrika 83, $715-726$.

Branco, M.; Dey, D. (2001). A general class of multivariate elliptical distributions. $J$. Multivariate Anal. 79, 99-113.

Gupta, R.C.; Brown, N. (2001). Reliability studies of the skew-normal distribution and its application to a strength-stress model. Comm. Statist. Theory Methods 30, 2427-2445.

Gupta, R.C.; Gupta, R.D. (2004). Generalized skew normal density. Test 13, 501-524.

Henze, N. (1986). A probabilistic representation of the skew-normal distribution. Scand. J. Statist. 13, 271-275.

Karlin, S. (1968). Total Positivity, vol. 1. Stanford University Press, Stanford, CA.

Kotz, S.; Balakrishnan, N.; Johnson, N.L. (2000). Continuous Multivariate Distributions, vol. 1, 2nd ed. Wiley, New York.

Loperfido, N. (2001). Quadratic forms of skew-normal random vectors, Statist. Probab. Lett. 54, 381-387.

Sharafi, M.; Behboodian, J. (2006). The Balakrishnan skew-normal density. Statist. Papers, to appear. 


\section{Appendix A1}

Let $\Phi_{1}(z ; \theta)$ be the distribution function of $Z_{\theta} \sim \mathrm{SN}(\theta)$, and $U \sim \mathrm{N}(0,1)$. Then

$$
E\left\{\Phi^{2}(\lambda U+\lambda t)\right\}=\Phi_{1}\left(\frac{\lambda t}{\sqrt{1+\lambda^{2}}} ; \frac{1}{\sqrt{1+2 \lambda^{2}}}\right)
$$

Proof. Consider $U, U_{1}, U_{2}$ i.i.d $\mathrm{N}(0,1)$,

$$
\begin{aligned}
E\left\{\Phi^{2}(\lambda U+\lambda t)\right\} & =\int_{-\infty}^{\infty} \Phi^{2}(\lambda u+\lambda t) \varphi(u) d u \\
& =\int_{-\infty}^{\infty} P\left(U_{1} \leqslant \lambda U+\lambda t, U_{2} \leqslant \lambda U+\lambda t \mid U=u\right) \varphi(u) d u \\
& =P\left(U_{1} \leqslant \lambda U+\lambda t, U_{2} \leqslant \lambda U+\lambda t\right) \\
& =P\left(W_{1} \leqslant \frac{\lambda t}{\sqrt{1+\lambda^{2}}}, W_{2} \leqslant \frac{\lambda t}{\sqrt{1+\lambda^{2}}}\right) \\
& =P\left\{\max \left(W_{1}, W_{2}\right) \leqslant \frac{\lambda t}{\sqrt{1+\lambda^{2}}}\right\},
\end{aligned}
$$

where $W_{i}=\left(U_{i}-\lambda U\right) / \sqrt{1+\lambda^{2}}, i=1,2$, and $\left(W_{1}, W_{2}\right) \sim \mathrm{N}_{2}\left(0,0,1,1, \frac{\lambda^{2}}{1+\lambda^{2}}\right)$. By Loperfido (2001) we have

$$
\max \left\{W_{1}, W_{2}\right\} \sim \mathrm{SN}\left(\frac{1}{\sqrt{1+2 \lambda^{2}}}\right) .
$$

Thus, we have (16).

\section{Appendix A2}

Proof of the formulas (12) and (14): We know that

$$
E\left(X_{\lambda}^{k}\right)=\frac{d^{k}}{d t^{k}} M_{X_{\lambda}}(t) \mid t=0 .
$$

Therefore

$$
\begin{aligned}
E\left(X_{\lambda}\right)= & \left.\frac{d}{d t} M_{X_{\lambda}}(t)\right|_{t=0} \\
= & \frac{\pi}{\tan ^{-1} \sqrt{1+2 \lambda^{2}}}\left[t \exp \left\{\frac{t^{2}}{2}\right\} \Phi_{1}\left(\frac{\lambda t}{\sqrt{1+\lambda^{2}}} ; \frac{1}{\sqrt{1+2 \lambda^{2}}}\right)\right. \\
& \left.+\exp \left\{\frac{t^{2}}{2}\right\} \frac{d}{d t} \Phi_{1}\left(\frac{\lambda t}{\sqrt{1+\lambda^{2}}} ; \frac{1}{\sqrt{1+2 \lambda^{2}}}\right)\right]\left.\right|_{t=0}
\end{aligned}
$$


Becalise of

$\frac{d}{d t} \Phi_{1}\left(\frac{\lambda t}{\sqrt{1+\lambda^{2}}} ; \frac{1}{\sqrt{1+2 \lambda^{2}}}\right)=\frac{\lambda}{\sqrt{1+\lambda^{2}}} 2 \varphi\left(\frac{\lambda t}{\sqrt{1+\lambda^{2}}}\right) \Phi\left(\frac{\lambda t}{\sqrt{1+\lambda^{2}} \sqrt{1+2 \lambda^{2}}}\right)$,

we have

$$
\mu_{\lambda}=E\left(X_{\lambda}\right)=\left(\frac{\pi}{\tan ^{-1} \sqrt{1+2 \lambda^{2}}}\right)\left(\frac{\lambda}{\sqrt{2 \pi} \sqrt{1+\lambda^{2}}}\right) .
$$

Therefore the formula (12) is proved.

For $E\left(X_{\lambda}^{3}\right)$, by taking derivative and some calculations we obtain

$$
\begin{aligned}
E\left(X_{\lambda}^{3}\right)= & \left.\frac{d^{3}}{d t^{3}} M_{X_{\lambda}}(t)\right|_{t=0} \\
= & 3 \frac{d}{d t} \Phi_{1}\left(\frac{\lambda t}{\sqrt{1+\lambda^{2}}} ; \frac{1}{\sqrt{1+2 \lambda^{2}}}\right)+\left.\frac{d^{3}}{d t^{3}} \Phi_{1}\left(\frac{\lambda t}{\sqrt{1+\lambda^{2}}} ; \frac{1}{\sqrt{1+2 \lambda^{2}}}\right)\right|_{t=0} \\
= & 3\left(\frac{\lambda}{\tan ^{-1} \sqrt{1+2 \lambda^{2}}}\right)\left(\frac{\lambda}{\sqrt{2 \pi} \sqrt{1+\lambda^{2}}}\right) \\
& -\left(\frac{\pi}{\tan ^{-1} \sqrt{1+2 \lambda^{2}}}\right)\left(\frac{\lambda^{3}}{\sqrt{2 \pi}\left(1+\lambda^{2}\right) \sqrt{1+\lambda^{2}}}\right) \\
= & \left(\frac{\pi}{\tan ^{-1} \sqrt{1+2 \lambda^{2}}}\right)\left(\frac{\lambda}{\sqrt{2 \pi} \sqrt{1+\lambda^{2}}}\right)\left(3-\frac{\lambda^{2}}{1+\lambda^{2}}\right) \\
= & \left(\frac{3+2 \lambda^{2}}{1+\lambda^{2}}\right) \mu_{\lambda} .
\end{aligned}
$$

\section{Appendix A3}

Proof of some properties (6)-(11):

Proof of (6). For $k=1$ (see Azzalini, 1985),

$$
\begin{aligned}
E\left(X_{\lambda}\right)-E\left(Z_{\lambda}\right) & =\left(\frac{\pi}{\tan ^{-1} \sqrt{1+2 \lambda^{2}}}\right)\left(\frac{\lambda}{\sqrt{2 \pi} \sqrt{1+\lambda^{2}}}\right)-\sqrt{\frac{2}{\pi}}\left(\frac{\lambda}{\sqrt{1+\lambda^{2}}}\right) \\
& =\sqrt{\frac{2}{\pi}}\left(\frac{\lambda}{\sqrt{1+\lambda^{2}}}\right)\left(\frac{\pi}{2 \tan ^{-1} \sqrt{1+2 \lambda^{2}}}-1\right) .
\end{aligned}
$$

Because $\left\{\pi /\left(2 \tan ^{-1} \sqrt{1+2 \lambda^{2}}\right)\right\}-1 \geqslant 0$ for $\lambda \in \mathbb{R}$, we can see that

$$
E\left(X_{\lambda}\right) \geqslant E\left(Z_{\lambda}\right) \geqslant 0 \text { for } \lambda \geqslant 0
$$

and

$$
E\left(X_{\lambda}\right) \leqslant E\left(Z_{\lambda}\right) \leqslant 0 \text { for } \lambda \leqslant 0
$$


Now consider $k=3$. By (14) and Azzalini (1985),

$$
\begin{aligned}
E\left(X_{\lambda}^{3}\right)-E\left(Z_{\lambda}^{3}\right) & =\left(\frac{3+2 \lambda^{2}}{1+\lambda^{2}}\right) E\left(X_{\lambda}\right)-\left(\frac{3+2 \lambda^{2}}{1+\lambda^{2}}\right) E\left(Z_{\lambda}\right) \\
& =\left(\frac{3+2 \lambda^{2}}{1+\lambda^{2}}\right)\left\{E\left(X_{\lambda}\right)-E\left(Z_{\lambda}\right)\right\}
\end{aligned}
$$

By the above result for $E\left(X_{\lambda}\right)-E\left(Z_{\lambda}\right)$, we obtain (6) for $k=3$.

Proof of (7). By (13) and Azzalini (1985),

$$
\begin{aligned}
E\left(X_{\lambda}^{2}\right)-E\left(Z_{\lambda}^{2}\right) & =1+\left\{\frac{\lambda^{2}}{\left(1+\lambda^{2}\right) \sqrt{1+2 \lambda^{2}} \tan ^{-1} \sqrt{1+2 \lambda^{2}}}\right\}-1 \\
& =\frac{\lambda^{2}}{\left(1+\lambda^{2}\right) \sqrt{1+2 \lambda^{2}} \tan ^{-1} \sqrt{1+2 \lambda^{2}}} \geqslant 0 .
\end{aligned}
$$

Therefore $E\left(X_{\lambda}^{2}\right) \geqslant E\left(Z_{\lambda}^{2}\right)$ for $\lambda \in \mathbb{R}$.

\section{Appendix A4}

Proof of $\mu_{T}$ and $\sigma_{T}^{2}$ :

$$
\begin{aligned}
\mu_{T} & =E(T) \\
& =E\left(\frac{1}{n} \sum_{i=1}^{n} \frac{X_{i}}{\Phi\left(\lambda X_{i}\right)}\right) \\
& =E\left(\frac{X}{\Phi(\lambda X)}\right) \\
& =\int\left(\frac{\pi}{\tan ^{-1} \sqrt{1+2 \lambda^{2}}}\right)\left(\frac{x}{\Phi(\lambda x)}\right) \varphi(x) \Phi^{2}(\lambda x) d x \\
& =\left(\frac{\pi}{\tan ^{-1} \sqrt{1+2 \lambda^{2}}}\right) E\left(Z_{\lambda}\right) \\
& =\sqrt{\frac{\pi}{2}}\left(\frac{\lambda}{\sqrt{1+\lambda^{2}}}\right)\left(\frac{1}{\tan ^{-1} \sqrt{1+2 \lambda^{2}}}\right) .
\end{aligned}
$$


The variance of $T$ is

$$
\begin{aligned}
\sigma_{T}^{2} & =\operatorname{var}(T) \\
& =\frac{1}{n} \operatorname{var}\left(\frac{X}{\Phi(\lambda X)}\right) \\
& =\frac{1}{n}\left\{\int\left(\frac{\pi}{\tan ^{-1} \sqrt{1+2 \lambda^{2}}}\right)\left(\frac{x^{2}}{\Phi^{2}(\lambda x)}\right) \varphi(x) \Phi^{2}(\lambda x) d x-\mu_{T}^{2}\right\} \\
& =\frac{\pi}{n \tan ^{-1} \sqrt{1+2 \lambda^{2}}}\left(1-\mu_{T}^{2}\right) \\
& =\frac{\pi}{n \tan ^{-1} \sqrt{1+2 \lambda^{2}}}\left(1-\frac{\lambda^{2}}{2\left(1+\lambda^{2}\right) \tan ^{-1} \sqrt{1+2 \lambda^{2}}}\right) .
\end{aligned}
$$

\section{Maryam Sharafi}

Department of Statistics,

Shiraz University,

Shiraz, Iran.

e-mail: msharafi@susc.ac.ir

\section{Javad Behboodian}

Department of Mathematics,

Islamic Azad University, Shiraz Branch

Shiraz, Iran.

e-mail:behboodian@stat.susc.ac.ir 
